\title{
Scalar Curvature via Local Extent
}

https://doi.org/10.1515/agms-2018-0008

Received February 19, 2018; revised August 18, 2018; accepted September 24, 2018

Abstract: We give a metric characterization of the scalar curvature of a smooth Riemannian manifold, analyzing the maximal distance between $(n+1)$ points in infinitesimally small neighborhoods of a point. Since this characterization is purely in terms of the distance function, it could be used to approach the problem of defining the scalar curvature on a non-smooth metric space. In the second part we will discuss this issue, focusing in particular on Alexandrov spaces and surfaces with bounded integral curvature.

Keywords: Scalar curvature, $q$-extent, surfaces with bounded integral curvature, Alexandrov spaces MSC: 53C20, 53C23 (Primary) 53C45 (Secondary)

\section{Introduction}

It is well known that the volume growth of the geodesic balls of an $n$-dimensional Riemannian manifold $(M, g)$ is tightly related to curvature. On the one hand, the first non-trivial term of the asymptotic expansion of the volumes of infinitesimally small balls centered at a point $x \in M$ is given, up to a constant, by the scalar curvature at $x$. Namely one has

$$
\operatorname{Vol} g\left(B_{\varepsilon}^{M}(x)\right)=\operatorname{Vol}\left(B_{\varepsilon}^{\mathbb{R}^{n}}\right)\left(1-\frac{1}{6(n+2)} \operatorname{Scal}_{g}(x) \varepsilon^{2}+o\left(\varepsilon^{2}\right)\right),
$$

see for instance [12]. On the other hand, thanks to the celebrated Bishop-Gromov volumes comparison theorem, complete manifolds with globally lower bounded Ricci (or sectional) curvature, enjoy an upper control on the volume growth of their geodesic balls, i.e. for all $x \in M$ and $r \geq 0$

$$
\operatorname{Ric}_{g} \geq(n-1) K \Rightarrow \operatorname{Vol}_{g}\left(B_{r}^{M}(x)\right) \leq \operatorname{Vol}\left(B_{r}^{S_{K}^{n}}(x)\right),
$$

$S_{K}^{n}$ being the simply connected $n$-dimensional space form of constant sectional curvature $K$. Moreover, the equality in Bishop-Gromov is realized if and only if $B_{r}^{M}(x)$ and $B_{r}^{S_{K}^{n}}(x)$ are isometric. This latter characterization of the equality case can be seen as an extremal result for the recognition problem. Namely, a recognition problem in Riemannian geometry "asks for the identification of an unknown riemannian manifold via measurements of metric invariants on the manifold", [11]. In their program intended to attack extremal recognition problems, K. Grove and S. Markvorsen introduced a new metric invariant of a metric space $\left(X, d_{X}\right)$, which they called the $q$-extent. This roughly measures how far $q$-points of the space $X$ can be one from the others. Namely, one defines

$$
\mathrm{xt}_{q}(X):=\left(\begin{array}{c}
q \\
2
\end{array}\right)^{-1} \sup _{\left(x_{1}, \ldots, x_{q}\right) \in X^{q}} \sum_{1 \leq i<j \leq q} d_{X}\left(x_{i}, x_{j}\right),
$$

where the supremum is obviously a maximum when $X$ is compact. In this case, following [11], we call $q$ extender the set of $q$ points realizing the $q$-extent. Note that the $q$-extent naturally generalizes the diameter (i.e. the 2-extent) of the metric space and it is intimately related to the $q$-th packing radius

$$
\operatorname{pack}_{q} X:=\frac{1}{2} \max _{\left(x_{1}, \ldots, x_{q}\right) \in X^{q}} \min _{1 \leq i<j \leq q} d_{X}\left(x_{i}, x_{j}\right) ;
$$

*Corresponding Author: Giona Veronelli: Université Paris 13, Sorbonne Paris Cité, LAGA, CNRS (UMR 7539) 99 avenue JeanBaptiste Clément F-93430 Villetaneuse, France, E-mail: veronelli@math.univ-paris13.fr 
$[10,11]$. One of the purposes of Grove and Markvorsen's recognition program was to study the relation between the $q$-extent and a global lower bound on the sectional curvature of Riemannian or Alexandrov metric spaces. A (particular case of) a result of [11] says that an $n$-dimensional, $n \geq 2$, Alexandrov space $\left(X, d_{X}\right)$ with curvature (in the sense of Alexandrov) greater or equal to 1 satisfies

$$
\mathrm{xt}_{n+1}(X) \leq \mathrm{xt}_{n+1}\left(\mathbb{S}^{n}\right),
$$

$\mathbb{S}^{n}$ being the unit sphere of $\mathbb{R}^{n+1}$ with its canonical metric. Moreover, the equality is realized in (1.2) if and only if diam $X=\pi$, which, together with Toponogov's diameter sphere theorem, implies that $X$ is necessarily isometric to $\mathbb{S}^{n}$ provided it is a Riemannian manifold. ${ }^{1}$

According to what said above, it is then natural to expect that, as in the case of the volume of geodesic balls, the first nontrivial term in the asymptotic expansion of the $(n+1)$-extent of infinitesimally small geodesic balls centered at a point $x \in M$ involves the scalar curvature at $x$. This is the content of the next result.

Theorem 1.1. Let $(M, g)$ be a smooth $n$-dimensional Riemannian manifold and $x \in \operatorname{int}(M)$. Then

$$
\begin{aligned}
\mathrm{xt}_{n+1}\left(\bar{B}_{\varepsilon}^{M}(x)\right) & =\mathrm{xt}_{n+1}\left(\bar{B}_{1}^{\mathbb{R}^{n}}\right) \varepsilon-\frac{1}{6} \sqrt{\frac{n+1}{2 n^{5}}} \operatorname{Scal}_{g}(x) \varepsilon^{3}+\mathcal{O}\left(\varepsilon^{4}\right) \\
& =\sqrt{2 \frac{n+1}{n}} \varepsilon\left\{1-\frac{1}{12 n^{2}} \operatorname{Scal}_{g}(x) \varepsilon^{2}+\mathcal{O}\left(\varepsilon^{3}\right)\right\},
\end{aligned}
$$

as $\varepsilon \rightarrow 0$.

Since

$$
\mathrm{xt}_{n+1}\left(\bar{B}_{1}^{\mathbb{R}^{n}}\right) \varepsilon=\mathrm{xt}_{n+1}\left(\bar{B}_{\varepsilon}^{\mathbb{R}^{n}}\right)=\varepsilon \sqrt{\frac{2(n+1)}{n}},
$$

see Lemma 2.1 below, we trivially deduce

Corollary 1.2. Let $(M, g)$ be a smooth $n$-dimensional Riemannian manifold and $x \in \operatorname{int}(M)$. Then

$$
\operatorname{Scal}_{g}(x)=\lim _{\varepsilon \rightarrow 0} \frac{12 n^{2}}{\varepsilon^{2}}\left\{1-\frac{\mathrm{xt}_{n+1}\left(\bar{B}_{\varepsilon}^{M}(x)\right)}{\mathrm{xt}_{n+1}\left(\bar{B}_{\varepsilon}^{\mathbb{R}^{n}}\right)}\right\} .
$$

From here on, given a metric space $(X, d)$, we will denote the closed metric ball $\{y \in X: d(x, y) \leq \epsilon\}$ either by $\bar{B}_{\varepsilon}^{X}(x)$ (whenever the metric acting on the space $X$ is implicitly understood) or by $\bar{B}_{\varepsilon}^{d}(x)$ (whenever the space $X$ on which the metric $d$ acts is implicitly understood).

The main ingredients in the proof of Theorem 1.1 are

- An asymptotic formula for the distance function in geodesic normal coordinates, [8, 9]. On the tangent space at $x$, one can consider two different distances between two vectors $u$ and $v$ : the Euclidean one $|u-v|$ and the distance induced by the Riemannian metric $g$ pulled-back via the exponential map, i.e. $d_{g}\left(\exp _{x}(u), \exp _{x}(v)\right)$. Their difference is given (at the first nontrivial order) by $R_{g}(u, v, v, u)$, where $R_{g}$ is the Riemann tensor of $g$; see Lemma 2.5 below.

- According to [18], the only $(n+1)$-extenders in the $n$-dimensional Euclidean ball are the regular simplexes. Here we need a quantitative analysis characterizing the $(n+1)$-simplexes which almost realize the extent; see Proposition 2.2 below.

- By its very definition it turns out that the scalar curvature is (twice) the average of the sectional curvatures of all the planes spanned by an orthonormal frame. The same holds true if instead one considers all the planes spanned by the vertices of a regular $(n+1)$-simplex; see Lemma 2.6.

In view of possible applications to metric spaces, some pathological behavior can arise in the $(n+1)$ extenders of geodesic balls. For instance, for thin 2-dimensional cones, the 3-extent of a metric ball around

1 Further results involving the $q$-extent can be found in [15, 26] or the survey [20]. 
the vertex is realized by 3 points including the vertex itself. In some non-rigorous sense the extender's shape is thus discontinuous with respect to the width of the angle. For this reason, we are led to introduce a slightly modified object, which we call boundary $(n+1)$-extent and which can be defined for a closed geodesic ball $\bar{B}_{\epsilon}^{M}$ as

$$
\partial \mathrm{xt}_{n+1}\left(\bar{B}_{\epsilon}^{M}\right):=\left(\begin{array}{c}
n+1 \\
2
\end{array}\right)^{-1} \sup _{\left(x_{1}, \ldots, x_{q}\right) \in\left(\partial B_{\epsilon}\right)^{q}} \sum_{1 \leq i<j \leq q} d_{M}\left(x_{i}, x_{j}\right) .
$$

As it is clear from the proof, the Riemannian characterization of the scalar curvature given in Theorem 1.1 and Corollary 1.2 holds without changes if one replaces in the formulas the $(n+1)$-extent of the geodesic balls with their boundary $(n+1)$-extent. In particular we have the following

Theorem 1.3. Let $(M, g)$ be a smooth $n$-dimensional Riemannian manifold and $x \in \operatorname{int}(M)$. Then

$$
\operatorname{Scal}_{g}(x)=\lim _{\varepsilon \rightarrow 0} \frac{12 n^{2}}{\varepsilon^{2}}\left\{1-\frac{\partial \mathrm{xt}_{n+1}\left(\bar{B}_{\varepsilon}^{M}(x)\right)}{\partial \mathrm{xt}_{n+1}\left(\bar{B}_{\varepsilon}^{\mathbb{R}^{n}}\right)}\right\} .
$$

Clearly, as in Corollary 1.2, also the formula (1.1), as well as the well-known similar expansion for the area of the surface of small geodesic balls, allows alternative definitions of the scalar curvature at $x \in M$ depending on the geometry of (arbitrarily small) neighborhood of $x$. However, the characterization of the scalar curvature expressed via the $(n+1)$-extent has the peculiarity of depending explicitely on the sole distance function of $M$, and not on the whole Riemannian structure. By a theoretical point of view, this advantage is negligible since a) the distance function uniquely determines the Riemannian metric tensor, [21], and b) whenever the volume measure of the underlying space is given by its $n$-dimensional Hausdorff measure, also the volume measure depends on the distance function. However, the explicit dependence given in (1.3) seems nontrivial. This suggests that (1.3) could be used to give a definition of scalar curvature (bounds) of a metric space.

In this direction, we will propose some possible approaches. First, (1.3) can be used as it is to introduce a notion of point-wise defined (dimensional) scalar curvature on a metric space $(X, d)$. The asymptotic limit in (1.3) in general will not exist, however a lim inf (resp. lim sup) version can still be used to define spaces with lower (resp. upper) bounded scalar curvature, see Definition 3.1 and 3.2 below. This notion of metric scalar curvature reveals consistent at least on metric spaces with lower bounded curvature in the sense of Alexandrov (on which a unique natural concept of integer dimension is defined). In fact, we have the following result. The inequality part was already observed in [11, formula (7)], while the rigidity is proven in Proposition 3.4 below.

Theorem 1.4. Let $(X, d)$ be a n-dimensional $C B B(k)$ Alexandrov space, for some $k \in \mathbb{R}$. Then for all $x \in X$ and $\epsilon>0$,

$$
\mathrm{xt}_{n+1}\left(\bar{B}_{\varepsilon}^{X}(x)\right) \leq \mathrm{xt}_{n+1}\left(\bar{B}_{\varepsilon}^{S_{k}^{n}}\right),
$$

where $S_{k}^{n}$ is the simply connected $n$-dimensional space form of constant curvature $k$.

Moreover, if either $\epsilon \leq \frac{\pi}{4 \sqrt{k}}$ or $k \leq 0$, then equality holds in (1.5) if and only if $\bar{B}_{\varepsilon}^{X}(x)$ contains an isometric copy of $\Delta_{k}^{n+1}(\epsilon)$ with totally geodesic interior. Here, $\Delta_{k}^{n+1}(\epsilon)$ is the unique (up to isometries) regular $(n+1)$-simplex inscribed in $\bar{B}_{\varepsilon}^{S_{k}^{n}}$.

A special class of two dimensional metric spaces is given by the surfaces with bounded integral curvature introduced by Alexandrov. These spaces have a natural notion of curvature measure, which can be described for instance as a weak limit of integral curvatures along an approximating sequence of smooth Riemannian metrics (for details, see Section 4 and the references therein). In Proposition 4.1 we will show that the regular part of this curvature measure can be point-wisely obtained via the local extent as in (1.3). Clearly on the support of the singular part of the curvature measure, the 3 -extent of geodesic $\varepsilon$-balls does not converge as $\varepsilon \rightarrow 0$, so that a point-wise definition is there ill-posed.

Because of the intrinsic singularity of a general Alexandrov space, it would be more natural to define on it a scalar curvature measure instead of a point-wise defined scalar curvature function. I'm grateful to J. 
Bertrand and M. Gromov for pointing this out to me. In the interesting paper [17], Kapovitch, Lytchak and Petrunin proposed a notion of mm-curvature measure which constitute a more general measure version of the asymptotic characterization (1.1). In particular they proved that such a measure is well-defined on BIC surfaces, although unfortunately it does not coincide with the canonical intrinsic curvature measure. In Section 5 we will propose a construction similar to that of [17], giving on general BIC surfaces a measure version of the characterizations of the scalar curvature via both the expansion (3.1) of the extent and the expansion (3.2) of the boundary extent of geodesic balls.

Indeed, consider for the moment a smooth $n$-dimensional Riemannian manifold $(M, g)$. For some $r_{0}>0$ small enough let us define two families of measure $\left\{e_{r}\right\}_{0<r<r_{0}}$ and $\left\{\partial e_{r}\right\}_{0<r<r_{0}}$ by

$$
e_{r}:=\left(1-\frac{\mathrm{xt}_{n+1}\left(B_{r}(x)\right)}{\mathrm{xt}_{n+1}\left(\bar{B}_{r}^{\mathbb{R}^{n}}\right)}\right) \mathcal{H}^{n}
$$

and

$$
\partial e_{r}:=\left(1-\frac{\partial \mathrm{xt}_{n+1}\left(\bar{B}_{r}(x)\right)}{\partial \mathrm{xt}_{n+1}\left(\bar{B}_{r}^{\mathbb{R}^{n}}\right)}\right) \mathcal{H}^{n} .
$$

In view of Theorem 1.1 and 1.3 we have that both $r^{-2} e_{r}$ and $r^{-2} \partial e_{r}$ converge weakly in the sense of measure to $\mathrm{Scal}_{g} \cdot \mathcal{H}^{n}$ as $r \rightarrow 0$. On a non-smooth surface with bounded integral curvature we can still prove that the families $\left\{r^{-2} e_{r}\right\}$ and $\left\{r^{-2} \partial e_{r}\right\}$ are uniformly bounded, so that at least some subsequence converges; see Theorem 5.1.

The paper is organized as follows. In Section 2 we prove the Riemannian characterization given by Theorem 1.1 and propose a possible generalization involving the $p$ powers of the distances, see Theorem 2.8. In Section 3 we consider the point-wise definition of (bounds on the) scalar curvature on Alexandrov spaces. In the last two sections we focus on surfaces with bounded integral curvature. In Section 4 we show that the point-wise characterization holds on the regular part of BIC surfaces, while in Section 5 we introduce the (boundary) extent curvature measures inspired by the construction of [17].

\section{Proof of the Riemannian characterization}

Before starting the proof of Theorem 1.1, let us remark that the result is trivial for $M=\mathbb{R}^{n}$. In this case, the value of $\mathrm{xt}_{n+1}\left(\bar{B}_{1}^{\mathbb{R}^{n}}\right)$ is given by the following lemma; see [18, Lemma 3].

Lemma 2.1. It holds

$$
\mathrm{xt}_{n+1}\left(\bar{B}_{1}^{\mathbb{R}^{n}}\right)=\sqrt{2 \frac{n+1}{n}} .
$$

Moreover the regular $(n+1)$-simplexes inscribed in $\bar{B}_{1}^{\mathbb{R}^{n}}$ are the only $(n+1)$-tuples of points realizing the equality.

We recall here some basic useful facts concerning regular simplexes in the Euclidean space. Fix $\left\{e_{i}\right\}_{i=1}^{n}$ the canonical orthonormal basis of $\mathbb{R}^{n}$. For $p=1, \ldots, n$ and for $k=1, \ldots, p+1$, we introduce the vectors $S_{k}^{p} \in \mathbb{R}^{n}$ defined by $S_{1}^{p}=e_{p}$ for $p=1, \ldots, n$ and by the inductive rule

$$
S_{k}^{p}=-\frac{1}{p} S_{1}^{p}+\frac{\sqrt{p^{2}-1}}{p} S_{k-1}^{p-1}
$$

It is easy to see that

$$
\mathcal{S}^{n}=\left\{S_{k}^{n}\right\}_{k=1}^{n+1} \in\left(\mathbb{R}^{n}\right)^{n+1}
$$

is a regular $(n+1)$-simplex inscribed in $\partial B_{1}^{\mathbb{R}^{n}}$. Similarly, for any $1 \leq p \leq n$, the points $\left\{S_{j}^{p}\right\}_{j=1}^{p+1}$ form a regular $(p+1)$-simplex inscribed in $\partial B_{1}^{\mathbb{R}^{p}} \times\left\{0_{\mathbb{R}^{n-p}}\right\}$. For convenience we consider $\left\{S_{1}^{1}, S_{2}^{1}\right\}=\left\{e_{1},-e_{1}\right\}$ as a regular 2-simplex of $\mathbb{R}^{1} \times\left\{0_{\mathbb{R}^{n-1}}\right\}$.

In the following, we will need a stable version of the equality case in Lemma 2.1, that is, simplexes which approximate the equality are almost regular. From now on, following standard notation, we will say that a 
(possibly vector-valued) function $f:\left(0, \varepsilon_{0}\right) \rightarrow \mathbb{R}$ for some $\varepsilon_{0}>0$ satisfies $f(\varepsilon)=\mathcal{O}\left(\varepsilon^{a}\right)$ for some $a \in \mathbb{Z}$ if there exists a constant $C$ (possibly depending on the underlying manifold $M$ ) such that $\lim _{\sup } \rightarrow 0(\varepsilon) \mid \varepsilon^{-a} \leq C$.

As announced above, we have the following

Proposition 2.2. Let $\varepsilon \mapsto P_{\varepsilon, k} \in B_{1}^{\mathbb{R}^{n}}, k=1 \ldots n+1$, be $n+1$ vector valued functions defined on $\left(0, \varepsilon_{0}\right)$ for some small $\varepsilon_{0}>0$. If

$$
\sum_{1 \leq k<j \leq n+1}\left|P_{\varepsilon, k}-P_{\varepsilon, j}\right|-\sqrt{\frac{n(n+1)^{3}}{2}}=\mathcal{O}\left(\varepsilon^{2}\right), \quad \text { as } \varepsilon \rightarrow 0,
$$

then

$$
\left|P_{\varepsilon, k}-P_{\varepsilon, j}\right|-\sqrt{\frac{2(n+1)}{n}}=\mathcal{O}(\varepsilon), \quad \text { as } \varepsilon \rightarrow 0,
$$

for any $1 \leq k<j \leq n+1$. Moreover, fixed a regular $(n+1)$-simplex $\mathcal{S}^{n}=\left\{S_{k}^{n}\right\}_{k=1}^{n+1} \in\left(\mathbb{R}^{n}\right)^{n+1}$ inscribed in $\partial B_{1}^{\mathbb{R}^{n}}$, there exists a function $A:\left(0, \varepsilon_{0}\right) \rightarrow O(n)$ (taking values in the isometries group of $\left.\bar{B}_{1}^{\mathbb{R}^{n}}\right)$ such that

$$
\left|P_{\varepsilon, k}-A(\varepsilon) S_{k}^{n}\right|=\mathcal{O}(\varepsilon)
$$

as $\varepsilon \rightarrow 0$ for all $1 \leq k \leq n+1$.

Proof. Let $G$ be the barycenter of the given points, i.e. $G=\frac{1}{n+1} \sum_{k=1}^{n+1} P_{\varepsilon, k}$. Reasoning as in [18, Lemma 3], we get

$$
\begin{aligned}
\sum_{1 \leq k<j \leq n+1}\left|P_{\varepsilon, k}-P_{\varepsilon, j}\right|^{2} & =\sum_{1 \leq k<j \leq n+1}\left|P_{\varepsilon, k}-G\right|^{2}+\sum_{1 \leq k<j \leq n+1}\left|P_{\varepsilon, j}-G\right|^{2}-2 \sum_{1 \leq k<j \leq n+1}\left\langle P_{\varepsilon, k}-G, P_{\varepsilon, j}-G\right\rangle \\
& =n \sum_{k=1}^{n+1}\left|P_{\varepsilon, k}-G\right|^{2}-\left|\sum_{k=1}^{n+1}\left(P_{\varepsilon, k}-G\right)\right|^{2}+\sum_{k=1}^{n+1}\left|P_{\varepsilon, k}-G\right|^{2} \\
& =(n+1) \sum_{k=1}^{n+1}\left|P_{\varepsilon, k}-G\right|^{2} .
\end{aligned}
$$

Hence

$$
\begin{aligned}
\frac{n(n+1)^{3}}{2}-\frac{n(n+1)^{3}}{2}|G|^{2}-\frac{n(n+1)^{2}}{2} \sum_{k=1}^{n+1}\left(1-\left|P_{\varepsilon, k}\right|^{2}\right) & =\frac{n(n+1)^{2}}{2} \sum_{k=1}^{n+1}\left|P_{\varepsilon, k}\right|^{2}-\frac{n(n+1)^{3}}{2}|G|^{2} \\
& =\frac{n(n+1)^{2}}{2} \sum_{k=1}^{n+1}\left|P_{\varepsilon, k}-G\right|^{2} \\
& =\frac{n(n+1)}{2} \sum_{1 \leq k<j \leq n+1}\left|P_{\varepsilon, k}-P_{\varepsilon, j}\right|^{2} \\
& =\left(\sum_{1 \leq k<j \leq n+1}\left|P_{\varepsilon, k}-P_{\varepsilon, j}\right|\right)^{2} \frac{1}{\cos ^{2} \alpha},
\end{aligned}
$$

where $\alpha$ is the angle at the origin in $\mathbb{R}^{n(n+1) / 2}$ formed by the vector $E=\left(\left|P_{\varepsilon, k}-P_{\varepsilon, j}\right|\right)_{1 \leq k<j \leq n+1} \in \mathbb{R}^{n(n+1) / 2}$ and by the vector $I \in \mathbb{R}^{n(n+1) / 2}$ all of whose components are 1. Condition (2.3) ensures that $\cos \alpha \neq 0$ for $\varepsilon$ small enough and that

$$
\frac{n(n+1)^{2}}{2}\left[(n+1) \sin ^{2} \alpha+\cos ^{2} \alpha\left((n+1)|G|^{2}+\sum_{k=1}^{n+1}\left(1-\left|P_{\varepsilon, k}\right|^{2}\right)\right)\right] \leq \mathcal{O}\left(\varepsilon^{2}\right) .
$$

In particular $\alpha=\mathcal{O}(\varepsilon),|G|=\mathcal{O}(\varepsilon)$, and

$$
\left(1-\left|P_{\varepsilon, k}\right|^{2}\right)=\mathcal{O}\left(\varepsilon^{2}\right), \quad \forall k=1, \ldots, n+1 .
$$


Let $v \in \mathbb{R}$ be the constant such that $v I$ is the projection of $E$ onto the line spanned by $I$. Since $\alpha=\mathcal{O}(\varepsilon)$, we deduce that $|E-v I| \leq|v I| \tan \alpha=\mathcal{O}(\varepsilon)$, i.e. $\left|P_{\varepsilon, k}-P_{\varepsilon, j}\right|=v+\mathcal{O}(\varepsilon)$ for all $1 \leq k<j \leq n+1$. Assumption (2.3) implies $v=\sqrt{2 \frac{n+1}{n}}$.

We introduce vectors $P_{\varepsilon, k}^{p} \in \mathbb{R}^{n}$ for $p=1, \ldots, n$ and $k=1, \ldots, p+1$ which are defined as follows. For $p=n$ we set $P_{\varepsilon, k}^{n}:=P_{\varepsilon, k}$ for $k=1, \ldots, n+1$, while for $p<n$ they are defined inductively on $p$ by the same recursive relation as in (2.1), that is,

$$
P_{\varepsilon, k-1}^{p-1}:=\frac{p}{\sqrt{p^{2}-1}}\left(P_{\varepsilon, k}^{p}+\frac{1}{p} P_{\varepsilon, 1}^{p}\right), \quad k=2, \ldots, p+1 .
$$

Lemma 2.3. With notations above, we have that

$$
\left|P_{\varepsilon, 1}^{1}+P_{\varepsilon, 2}^{1}\right|=\mathcal{O}(\varepsilon) .
$$

Moreover for all $1 \leq k<p \leq n$ and for all $1 \leq j \leq k+1$ it holds

$$
\left\langle P_{\varepsilon, 1}^{p}, P_{\varepsilon, j}^{k}\right\rangle=\mathcal{O}(\varepsilon)
$$

and

$$
\left\langle P_{\varepsilon, 1}^{p}, P_{\varepsilon, j}^{p}\right\rangle= \begin{cases}-\frac{1}{p}+\mathcal{O}(\varepsilon), & \text { if } j>1, \\ 1+\mathcal{O}(\varepsilon), & \text { if } j=1,\end{cases}
$$

where $\langle\cdot, \cdot\rangle$ is the standard scalar product of $\mathbb{R}^{n}$.

Proof. The defining relations (2.6) imply

$$
\begin{aligned}
\sum_{j=1}^{p} P_{\varepsilon, j}^{p-1} & =\frac{p}{\sqrt{p^{2}-1}} \sum_{j=2}^{p+1}\left(P_{\varepsilon, j}^{p}+\frac{1}{p} P_{\varepsilon, 1}^{p}\right) \\
& =\frac{p}{\sqrt{p^{2}-1}}\left[\sum_{j=2}^{p+1}\left(P_{\varepsilon, j}^{p}\right)+P_{\varepsilon, 1}^{p}\right]=\frac{p}{\sqrt{p^{2}-1}} \sum_{j=1}^{p+1} P_{\varepsilon, j}^{p},
\end{aligned}
$$

for any $p=2, \ldots, n$. Since $|G|=\left|\sum_{j=1}^{n+1} P_{\varepsilon, j}^{n}\right|=\mathcal{O}(\varepsilon)$, applying recursively the latter relation we get (2.7).

To prove (2.8), first remark that

$$
\begin{aligned}
2\left\langle P_{\varepsilon, 1}^{n}, P_{\varepsilon, j+1}^{n}\right\rangle & =\left|P_{\varepsilon, 1}^{n}\right|^{2}+\left|P_{\varepsilon, j+1}^{n}\right|^{2}-\left|P_{\varepsilon, 1}^{n}-P_{\varepsilon, j+1}^{n}\right|^{2} \\
& =2(1+\mathcal{O}(\varepsilon))-\left(2 \frac{n+1}{n}+\mathcal{O}(\varepsilon)\right) \\
& =\frac{2}{n}+\mathcal{O}(\varepsilon),
\end{aligned}
$$

which in turn implies

$$
\begin{aligned}
\left\langle P_{\varepsilon, 1}^{n}, P_{\varepsilon, j}^{n-1}\right\rangle & =\frac{n}{\sqrt{n^{2}-1}}\left\langle P_{\varepsilon, 1}^{n}, P_{\varepsilon, j+1}^{n}+\frac{1}{n} P_{\varepsilon, 1}^{n}\right\rangle \\
& =\frac{\left|P_{\varepsilon, 1}^{n}\right|^{2}}{\sqrt{n^{2}-1}}+\frac{n\left\langle P_{\varepsilon, 1}^{n}, P_{\varepsilon, j+1}^{n}\right\rangle}{\sqrt{n^{2}-1}}=\mathcal{O}(\varepsilon) .
\end{aligned}
$$

Moreover, for all $k, q \in\{1, \ldots, n\}$ and all $1 \leq j \leq k+1$,

$$
\begin{aligned}
\left\langle P_{\varepsilon, 1}^{q}, P_{\varepsilon, j-1}^{k-1}\right\rangle & =\frac{k}{\sqrt{k^{2}-1}}\left\langle P_{\varepsilon, 1}^{q}, P_{\varepsilon, j}^{k}+\frac{1}{k} P_{\varepsilon, 1}^{k}\right\rangle \\
& =\frac{k}{\sqrt{k^{2}-1}}\left\langle P_{\varepsilon, 1}^{q}, P_{\varepsilon, j}^{k}\right\rangle+\frac{1}{\sqrt{k^{2}-1}}\left\langle P_{\varepsilon, 1}^{q}, P_{\varepsilon, 1}^{k}\right\rangle .
\end{aligned}
$$

Applying this latter recursively with $q=n$ and $k=p+1, \ldots, n$ gives, together with (2.11),

$$
\left\langle P_{\varepsilon, 1}^{n}, P_{\varepsilon, j}^{p}\right\rangle=\mathcal{O}(\varepsilon)
$$


for all $1 \leq p \leq n-1$ and all $1 \leq j \leq p$. Applying again (2.12) with $q=p$ and $k \leq n+1$, using also (2.13), gives (2.8). Finally, we prove (2.9) once again by induction on $p$. By (2.5) and (2.10), (2.9) is verified for $p=n$. Now, suppose that (2.9) is verified for some $p \leq n$. Then

$$
\begin{aligned}
\left\langle P_{\varepsilon, j-1}^{p-1}, P_{\varepsilon, k-1}^{p-1}\right\rangle & =\frac{p^{2}}{p^{2}-1}\left\langle P_{\varepsilon, j}^{p}+\frac{1}{p} P_{\varepsilon, 1}^{p}, P_{\varepsilon, k}^{p}+\frac{1}{p} P_{\varepsilon, 1}^{p}\right\rangle \\
& =\frac{p^{2}}{p^{2}-1}\left(\left\langle P_{\varepsilon, j}^{p}, P_{\varepsilon, k}^{p}\right\rangle+\frac{1}{p}\left(\left\langle P_{\varepsilon, j}^{p}, P_{\varepsilon, k}^{p}\right\rangle+\left\langle\frac{1}{p} P_{\varepsilon, 1}^{p}, \frac{1}{p} P_{\varepsilon, 1}^{p}\right\rangle\right)+\frac{1}{p^{2}}\left|P_{\varepsilon, 1}^{p}\right|^{2}\right) \\
& =\frac{p^{2}}{p^{2}-1}\left(\left\langle P_{\varepsilon, j}^{p}, P_{\varepsilon, k}^{p}\right\rangle-\frac{1}{p^{2}}+\mathcal{O}(\varepsilon)\right),
\end{aligned}
$$

so that by the inductive assumption

$$
\left\langle P_{\varepsilon, j-1}^{p-1}, P_{\varepsilon, k-1}^{p-1}\right\rangle= \begin{cases}-\frac{1}{p-1}+\mathcal{O}(\varepsilon), & \text { if } j \neq k, \\ 1+\mathcal{O}(\varepsilon), & \text { if } j=k .\end{cases}
$$

An application of the following Lemma with $p=n$ will conclude the proof of Proposition 2.2.

Lemma 2.4. For every $1 \leq p \leq n$, there exists an isometry $A_{p} \in O(n)$ of $B_{1}^{\mathbb{R}^{n}}$ satisfying

$$
P_{\varepsilon, j}^{q}=A_{p} S_{j}^{q}+\mathcal{O}(\varepsilon), \quad \forall 1 \leq q \leq p, \forall 1 \leq j \leq q+1 .
$$

Proof. We proceed by induction on $p$. Let $A_{1} \in O(n)$ be any isometry of $B_{1}^{\mathbb{R}^{n}}$ satisfying $A_{1} S_{1}^{n}=\frac{P_{\varepsilon, 1}^{1}}{\left|P_{\varepsilon, 1}^{1}\right|}$. According to (2.5), $P_{\varepsilon, 1}^{1}=A_{1} S_{1}^{1}+\mathcal{O}(\varepsilon)$. Using (2.7), we get

$$
P_{\varepsilon, 2}^{1}=-P_{\varepsilon, 1}^{1}+\mathcal{O}(\varepsilon)=-A_{1} S_{1}^{1}+\mathcal{O}(\varepsilon)=A_{1} S_{2}^{1}+\mathcal{O}(\varepsilon) .
$$

In particular (2.14) is satisfied for $p=1$.

Now, suppose that (2.14) is satisfied for some $1 \leq p<n$. Composing $A_{p}$ with a suitable further isometry $A_{p}^{\prime}$, we can find a new isometry $A_{p+1} \in O(n)$ such that (2.14) is satisfied for $p+1$ instead of $p$. Namely, recall that the vectors $A_{p} S_{j}^{q}$, with $1 \leq q \leq p$ and $1 \leq j \leq q+1$ are all contained in a $p$-dimensional hyperplane $\mathcal{H}_{p}$ of $\mathbb{R}^{n}$. Moreover $A_{p} S_{1}^{p+1} \in \mathcal{H}_{p}^{\perp}<\mathbb{R}^{n}$. Then one can take the isometry $A_{p}^{\prime} \in O(n)$ which fix $\mathcal{H}_{p}$ (i.e. $A_{p}^{\prime} \in I_{\mathbb{R}^{p}} \otimes O(n-p)$, where $\left.O(n-p) \subset \operatorname{Iso}\left(\mathcal{H}_{p}^{\perp}\right)\right)$ and such that the projection of $P_{\varepsilon, 1}^{p+1}$ onto the $(n-p)$-dimensional hyperplane $\mathcal{H}_{p}^{\perp}<\mathbb{R}^{n}$ is parallel to $A_{p}^{\prime} A_{p} S_{1}^{p+1}$. Explicitly one has that for each $\varepsilon$ there exists $A_{p}^{\prime} \in O(n)$ and a unique positive $\alpha$ such that

$$
A_{p}^{\prime} A_{p} S_{j}^{q}=A_{p} S_{j}^{q}, \quad \forall 1 \leq q \leq p, \forall 1 \leq j \leq q+1,
$$

and

$$
\begin{aligned}
\alpha A_{p}^{\prime} A_{p} S_{1}^{p+1} & =P_{\varepsilon, 1}^{p+1}-\pi_{\text {span }\left\{A_{p} S_{1}^{j}\right\}_{j=1}^{p}} P_{\varepsilon, 1}^{p+1} \\
& =P_{\varepsilon, 1}^{p+1}-\sum_{j=1}^{p}\left\langle P_{\varepsilon, 1}^{p+1}, A_{p} S_{1}^{j}\right\rangle A_{p} S_{1}^{j} .
\end{aligned}
$$

According to (2.14), using also (2.8) and (2.9), we deduce

$$
\begin{aligned}
\left|\alpha A_{p}^{\prime} A_{p} S_{1}^{p+1}-P_{\varepsilon, 1}^{p+1}\right| & =\left|\sum_{j=1}^{p}\left\langle P_{\varepsilon, 1}^{p+1}, P_{\varepsilon, 1}^{j}+\mathcal{O}(\varepsilon)\right\rangle\left(P_{\varepsilon, 1}^{j}+\mathcal{O}(\varepsilon)\right)\right| \\
& =\left|\sum_{j=1}^{p}\left\langle P_{\varepsilon, 1}^{p+1}, P_{\varepsilon, 1}^{j}\right\rangle P_{\varepsilon, 1}^{j}+\mathcal{O}(\varepsilon)\right| \\
& =\mathcal{O}(\varepsilon) .
\end{aligned}
$$

Again by (2.9) we have also that $\alpha=\alpha(\varepsilon)=1+\mathcal{O}(\varepsilon)$. Setting $A_{p+1}:=A_{p}^{\prime} A_{p}$, we have proved (2.14) for $p+1$, hence recursively for every $1 \leq p \leq n$. 
We now come back to the proof of the main theorem.

Proof (of Theorem 1.1). Fix a point $x_{0} \in M$ and let $\epsilon_{0}$ small enough so that for all $0<\varepsilon<\varepsilon_{0}, \phi_{\varepsilon}=\exp _{x_{0}}(\varepsilon \cdot)$ : $\bar{B}_{1}^{\mathbb{R}^{n}}(0) \rightarrow \bar{B}_{\varepsilon}^{M}\left(x_{0}\right)$ is a diffeomorphism.

For every $\varepsilon \in\left(0, \varepsilon_{0}\right)$, let $\left\{Q_{\varepsilon, j}\right\}_{j=1}^{n+1}$ be an $(n+1)$-extender of $\bar{B}_{\varepsilon}^{M}\left(x_{0}\right)$, i.e.

$$
\frac{n(n+1)}{2} \mathrm{xt}_{n+1}\left(\bar{B}_{\varepsilon}^{M}\left(x_{0}\right)\right)=\sum_{1 \leq k<j \leq n+1} d_{g}\left(Q_{\varepsilon, k}, Q_{\varepsilon, j}\right) .
$$

For $k=1, \ldots, n$, define $P_{\varepsilon, k}:=\phi_{\varepsilon}^{-1}\left(Q_{\varepsilon, k}\right)$.

We need the following

Lemma 2.5. Fix a point $x_{0} \in M$ and let $\epsilon_{0}$ small enough so that for all $0<\varepsilon<\varepsilon_{0}, \phi_{\varepsilon}=\exp _{x_{0}}(\varepsilon \cdot): \bar{B}_{1}^{\mathbb{R}^{n}}(0) \rightarrow$ $\bar{B}_{\varepsilon}^{M}\left(x_{0}\right)$ is a diffeomorphism. Then for every distinct points $p_{1}, p_{2} \in \bar{B}_{1}^{\mathbb{R}^{n}}(0)$ we have

$$
d_{g}\left(\phi_{\varepsilon}\left(p_{1}\right), \phi_{\varepsilon}\left(p_{2}\right)\right)=\varepsilon\left|p_{1}-p_{2}\right|-\frac{\varepsilon^{3}}{6\left|p_{1}-p_{2}\right|} R_{x_{0}}\left(p_{1}, p_{2}, p_{2}, p_{1}\right)+\mathcal{O}\left(\varepsilon^{4}\right),
$$

where $R_{x_{0}}$ is the Riemann tensor of $(M, g)$ at $x_{0}$. Here and on, $\mathbb{R}^{n}$ is canonically identified with $T_{x_{0}} M$.

Proof. This follows easily from the asymptotic formula

$$
d_{g}^{2}\left(\phi_{\varepsilon}\left(p_{1}\right), \phi_{\varepsilon}\left(p_{2}\right)\right)=\varepsilon^{2}\left|p_{1}-p_{2}\right|^{2}-\frac{\varepsilon^{4}}{3} R_{X_{0}}\left(p_{1}, p_{2}-p_{1}, p_{2}-p_{1}, p_{1}\right)+\mathcal{O}\left(\varepsilon^{5}\right),
$$

which can be proved via explicit computations; see for instance $[8,9]$.

Lemma 2.5 gives that

$$
\frac{n(n+1)}{2} \mathrm{xt}_{n+1}\left(\bar{B}_{\varepsilon}^{M}\left(x_{0}\right)\right)=\varepsilon \sum_{1 \leq k<j \leq n+1}\left|P_{\varepsilon, k}-P_{\varepsilon, j}\right|-\frac{\varepsilon^{3}}{6} \sum_{1 \leq k<j \leq n+1} \frac{R_{x_{0}}\left(P_{\varepsilon, k}, P_{\varepsilon, j}, P_{\varepsilon, j}, P_{\varepsilon, k}\right)}{\left|P_{\varepsilon, k}-P_{\varepsilon, j}\right|}+\mathcal{O}\left(\varepsilon^{4}\right) .
$$

Note that

$$
\begin{aligned}
\frac{R_{x_{0}}\left(P_{\varepsilon, k}, P_{\varepsilon, j}, P_{\varepsilon, j}, P_{\varepsilon, k}\right)}{\left|P_{\varepsilon, k}-P_{\varepsilon, j}\right|} & =\frac{R_{x_{0}}\left(P_{\varepsilon, k}-P_{\varepsilon, j}, P_{\varepsilon, j}, P_{\varepsilon, j}, P_{\varepsilon, k}-P_{\varepsilon, j}\right)}{\left|P_{\varepsilon, k}-P_{\varepsilon, j}\right|} \\
& =\frac{\operatorname{Sect}_{x_{0}}\left(P_{\varepsilon, k} \wedge P_{\varepsilon, j}\right)\left[\left|P_{\varepsilon, k}-P_{\varepsilon, j}\right|^{2}\left|P_{\varepsilon, j}\right|^{2}-\left\langle P_{\varepsilon, j}, P_{\varepsilon, k}-P_{\varepsilon, j}\right\rangle^{2}\right]}{\left|P_{\varepsilon, k}-P_{\varepsilon, j}\right|} \\
& \leq 2\left|\operatorname{Sect}_{x_{0}}\left(P_{\varepsilon, k} \wedge P_{\varepsilon, j}\right)\right|,
\end{aligned}
$$

since $P_{\varepsilon, k} \in \bar{B}_{1}^{\mathbb{R}^{n}}$ for all $k=1, \ldots, n$. We get in particular that

$$
\left|\mathrm{xt}_{n+1}\left(\bar{B}_{\varepsilon}^{M}\left(x_{0}\right)\right)-\varepsilon \frac{2}{n(n+1)} \sum_{1 \leq k<j \leq n+1}\right| P_{\varepsilon, k}-P_{\varepsilon, j}|| \leq \frac{1}{3}\left\|\operatorname{Sect}_{x_{0}}\right\| \varepsilon^{3}+\mathcal{O}\left(\varepsilon^{4}\right) .
$$

Now, let $\mathcal{S}^{n}$ be the regular $(n+1)$-simplex of $\mathbb{R}^{n}$ introduced in (2.2). Consider $n+1$ points $\left\{T_{\varepsilon, k}^{n}\right\}_{k=1}^{n+1}$ in $\bar{B}_{\varepsilon}^{M}\left(x_{0}\right)$ defined by $T_{\varepsilon, k}^{n}=\phi_{\varepsilon}\left(S_{k}^{n}\right)$. Again by Lemma 2.5, for every $1 \leq k<j \leq n+1$

$$
\begin{aligned}
d_{g}\left(T_{\varepsilon, k}^{n}, T_{\varepsilon, j}^{n}\right) & =\varepsilon\left|S_{k}^{n}-S_{j}^{n}\right|-\frac{\varepsilon^{3}}{6\left|S_{k}^{n}-S_{j}^{n}\right|} R_{x_{0}}\left(S_{k}^{n}, S_{j}^{n}, S_{j}^{n}, S_{k}^{n}\right)+\mathcal{O}\left(\varepsilon^{4}\right) \\
& =\varepsilon \sqrt{2 \frac{n+1}{n}}-\frac{\varepsilon^{3}}{6} \sqrt{\frac{n}{2(n+1)}} R_{x_{0}}\left(S_{k}^{n}, S_{j}^{n}, S_{j}^{n}, S_{k}^{n}\right)+\mathcal{O}\left(\varepsilon^{4}\right) .
\end{aligned}
$$


This latter, together with (2.18) and Lemma 2.1, yield

$$
\begin{aligned}
\sum_{1 \leq k<j \leq n+1}\left|S_{k}^{n}-S_{j}^{n}\right| & \leq \varepsilon^{-1} \sum_{1 \leq k<j \leq n+1} d_{g}\left(T_{\varepsilon, k}^{n}, T_{\varepsilon, j}^{n}\right)+\mathcal{O}\left(\varepsilon^{2}\right) \\
& \leq \varepsilon^{-1} \frac{n(n+1)}{2} \mathrm{xt}_{n+1}\left(\bar{B}_{\varepsilon}^{M}\left(x_{0}\right)\right)+\mathcal{O}\left(\varepsilon^{2}\right) \\
& \leq \sum_{1 \leq k<j \leq n+1}\left|P_{\varepsilon, k}-P_{\varepsilon, j}\right|+\mathcal{O}\left(\varepsilon^{2}\right) \\
& \leq \frac{n(n+1)}{2} \mathrm{xt}_{n+1}\left(\bar{B}_{1}^{\mathbb{R}^{n}}\right)+\mathcal{O}\left(\varepsilon^{2}\right) \\
& =\sum_{1 \leq k<j \leq n+1}\left|S_{k}^{n}-S_{j}^{n}\right|+\mathcal{O}\left(\varepsilon^{2}\right) .
\end{aligned}
$$

In particular,

$$
\sum_{1 \leq k<j \leq n+1}\left|P_{\varepsilon, k}-P_{\varepsilon, j}\right|=\sqrt{\frac{n(n+1)^{3}}{2}}+\mathcal{O}\left(\varepsilon^{2}\right) .
$$

We can thus apply Proposition 2.2 to deduce

$$
P_{\varepsilon, k}=A(\varepsilon) S_{k}^{n}+\mathcal{O}(\varepsilon), \quad \forall 1 \leq k \leq n+1 .
$$

for some function $A:\left(0, \varepsilon_{0}\right) \rightarrow O(n)$ taking values in the isometries group of $\bar{B}_{1}^{\mathbb{R}^{n}}$.

We are going to use the following Lemma, which will be proved later.

Lemma 2.6. Let $\mathcal{S}^{n}=\left\{S_{k}^{n}\right\}_{k=1}^{n+1} \in\left(\mathbb{R}^{n}\right)^{n+1}$ be any regular $(n+1)$-simplex inscribed in $\bar{B}_{1}^{\mathbb{R}^{n}}(0)$. Then

$$
\sum_{1 \leq k<j \leq n+1} R_{x_{0}}\left(S_{k}^{n}, S_{j}^{n}, S_{j}^{n}, S_{k}^{n}\right)=\frac{(n+1)^{2}}{2 n^{2}} \operatorname{Scal} g\left(x_{0}\right) .
$$

Since for $1 \leq k \leq n+1, P_{\varepsilon, k} \in \bar{B}_{1}^{\mathbb{R}^{n}}(0)$, Lemma 2.1 gives that

$$
\sum_{1 \leq k<j \leq n+1}\left|P_{\varepsilon, k}-P_{\varepsilon, j}\right| \leq \frac{n(n+1)}{2} \mathrm{xt}_{n+1}\left(\bar{B}_{1}^{\mathbb{R}^{n}}\left(x_{0}\right)\right)=\sum_{1 \leq k<j \leq n+1}\left|S_{k}^{n}-S_{j}^{n}\right| .
$$

Moreover, according to (2.21) and Lemma 2.6,

$$
\begin{aligned}
\sum_{1 \leq k<j \leq n+1} \frac{R_{x_{0}}\left(P_{\varepsilon, k}, P_{\varepsilon, j}, P_{\varepsilon, j}, P_{\varepsilon, k}\right)}{\left|P_{\varepsilon, k}-P_{\varepsilon, j}\right|} & =\sum_{1 \leq k<j \leq n+1} \frac{R_{x_{0}}\left(A(\varepsilon) S_{k}^{n}+\mathcal{O}(\varepsilon), A(\varepsilon) S_{j}^{n}+\mathcal{O}(\varepsilon), A(\varepsilon) S_{j}^{n}+\mathcal{O}(\varepsilon), A(\varepsilon) S_{k}^{n}+\mathcal{O}(\varepsilon)\right)}{\left|A(\varepsilon) S_{k}^{n}-A(\varepsilon) S_{j}^{n}\right|+\mathcal{O}(\varepsilon)} \\
& =\sum_{1 \leq k<j \leq n+1} \frac{R_{x_{0}}\left(A(\varepsilon) S_{k}^{n}, A(\varepsilon) S_{j}^{n}, A(\varepsilon) S_{j}^{n}, A(\varepsilon) S_{k}^{n}\right)}{\left|A(\varepsilon) S_{k}^{n}-A(\varepsilon) S_{j}^{n}\right|}+\mathcal{O}(\varepsilon) \\
& =\sqrt{\frac{n}{2(n+1)}} \sum_{1 \leq k<j \leq n+1} R_{x_{0}}\left(A(\varepsilon) S_{k}^{n}, A(\varepsilon) S_{j}^{n}, A(\varepsilon) S_{j}^{n}, A(\varepsilon) S_{k}^{n}\right)+\mathcal{O}(\varepsilon) \\
& =\sqrt{\frac{n}{2(n+1)}} \frac{(n+1)^{2}}{2 n^{2}} \operatorname{Scal}_{g}\left(x_{0}\right)+\mathcal{O}(\varepsilon) .
\end{aligned}
$$

Inserting these latter in (2.17) we get

$$
\frac{n(n+1)}{2} \mathrm{xt}_{n+1}\left(\bar{B}_{\varepsilon}^{M}\left(x_{0}\right)\right) \leq \varepsilon \sum_{1 \leq k<j \leq n+1}\left|S_{k}^{n}-S_{j}^{n}\right|-\frac{\varepsilon^{3}}{12} \sqrt{\frac{(n+1)^{3}}{2 n^{3}}} \operatorname{Scal}_{g}\left(x_{0}\right)+\mathcal{O}\left(\varepsilon^{4}\right)
$$

On the other hand, from (2.19) and Lemma 2.6, we have also

$$
\begin{aligned}
\frac{n(n+1)}{2} \mathrm{xt}_{n+1}\left(\bar{B}_{\varepsilon}^{M}\left(x_{0}\right)\right) & \geq \sum_{1 \leq k<j \leq n+1} d_{g}\left(T_{\varepsilon, k}^{n}, T_{\varepsilon, k}^{n}\right) \\
& =\varepsilon \sum_{1 \leq k<j \leq n+1}\left|S_{k}^{n}-S_{j}^{n}\right|-\frac{\varepsilon^{3}}{12} \sqrt{\frac{(n+1)^{3}}{2 n^{3}}} \operatorname{Scal}_{g}\left(x_{0}\right)+\mathcal{O}\left(\varepsilon^{4}\right) .
\end{aligned}
$$

The asymptotic estimates (2.23) and (2.24), together with Lemma 2.1, conclude the proof of Theorem 1.1. 
It remains to prove Lemma 2.6.

Proof (of Lemma 2.6). For $p=1, \ldots, n$ and $k=1, \ldots, p+1$, define unitary vectors $S_{k}^{p} \in \mathbb{R}^{n}$ as in (2.1), and fix an orthonormal basis of $T_{\chi_{0}} M=\mathbb{R}^{n}$ by setting $e_{j}=S_{1}^{j}$ for $1 \leq j \leq n$.

We are going to prove by induction on $n$ that

$$
\sum_{1 \leq k<j \leq n+1} R_{x_{0}}\left(S_{k}^{n}, S_{j}^{n}, S_{j}^{n}, S_{k}^{n}\right)=\frac{(n+1)^{2}}{n^{2}} \sum_{1 \leq k<j \leq n} \operatorname{Sect}_{x_{0}}\left(e_{k} \wedge e_{j}\right) .
$$

For the shortness of notation, for vectors $X, Y \in \mathbb{R}^{n}$, we set

$$
\kappa(X, Y):=R_{X_{0}}(X, Y, Y, X) .
$$

For $n=2$, for every $1 \leq k<j \leq 3$,

$$
\kappa\left(S_{k}^{2}, S_{j}^{2}\right)=\operatorname{Sect}_{x_{0}}\left(\left|S_{k}^{2}\right|^{2}\left|S_{j}^{2}\right|^{2}-\left\langle S_{k}^{2}, S_{j}^{2}\right\rangle^{2}\right)=\left(1-\frac{1}{4}\right) \operatorname{Sect}_{x_{0}}=\frac{3}{4} \operatorname{Sect}_{x_{0}},
$$

so that

$$
\sum_{1 \leq k<j \leq 3} \kappa\left(S_{k}^{2}, S_{j}^{2}\right)=\frac{9}{4} \operatorname{Sect}_{x_{0}}=\frac{9}{4} \operatorname{Sect}_{x_{0}}\left(e_{1}, e_{2}\right) .
$$

Lemma 2.7. For every $1 \leq p \leq n-1$, one has

$$
\sum_{k=1}^{p+1} \kappa\left(S_{1}^{n}, S_{k}^{p}\right)=\frac{p+1}{p} \sum_{j=1}^{p} \operatorname{Sect}_{x_{0}}\left(e_{n} \wedge e_{j}\right) .
$$

Proof. We prove this by induction on $p$. First, note that $\sum_{k=1}^{p} S_{k}^{p-1}=0$. Accordingly,

$$
\sum_{k=2}^{p+1} R_{\chi_{0}}\left(S_{1}^{n}, S_{1}^{p}, S_{k-1}^{p-1}, S_{1}^{n}\right)=0,
$$

and for every $1 \leq p<n$,

$$
\begin{aligned}
\sum_{k=1}^{p+1} \kappa\left(S_{1}^{n}, S_{k}^{p}\right) & =\kappa\left(S_{1}^{n}, S_{1}^{p}\right)+\sum_{k=2}^{p+1} \kappa\left(S_{1}^{n},-\frac{1}{p} S_{1}^{p}+\frac{\sqrt{p^{2}-1}}{p} S_{k-1}^{p-1}\right) \\
& =\kappa\left(S_{1}^{n}, S_{1}^{p}\right)+\frac{p}{p^{2}} \kappa\left(S_{1}^{n}, S_{1}^{p}\right)+\frac{p^{2}-1}{p^{2}} \sum_{k=2}^{p+1} \kappa\left(S_{1}^{n}, S_{k-1}^{p-1}\right) \\
& =\left(\frac{p+1}{p}\right) \kappa\left(S_{1}^{n}, S_{1}^{p}\right)+\frac{p^{2}-1}{p^{2}} \sum_{k=1}^{p} \kappa\left(S_{1}^{n}, S_{k}^{p-1}\right) \\
& =\left(\frac{p+1}{p}\right) \operatorname{Sect}_{x_{0}}\left(e_{n} \wedge e_{p}\right)+\frac{p^{2}-1}{p^{2}} \sum_{k=1}^{p} \kappa\left(S_{1}^{n}, S_{k}^{p-1}\right) .
\end{aligned}
$$

Since

$$
\sum_{k=1}^{2} \kappa\left(S_{1}^{n}, S_{k}^{1}\right)=2 \operatorname{Sect}_{x_{0}}\left(S_{1}^{n} \wedge S_{1}^{1}\right)=2 \operatorname{Sect}_{x_{0}}\left(e_{n} \wedge e_{1}\right),
$$

Lemma 2.7 is proved.

We come back to the proof of Lemma 2.6. Observe that, since $\sum_{j=2}^{n+1} S_{j-1}^{n-1}=0$ and by the linearity of the Riemann tensor, one get that

$$
\begin{aligned}
2 \sum_{2 \leq k<j \leq n+1} R_{x_{0}}\left(S_{1}^{n}, S_{k-1}^{n-1}, S_{j-1}^{n-1}, S_{1}^{n}\right) & =\sum_{2 \leq k<j \leq n+1}\left(R_{x_{0}}\left(S_{1}^{n}, S_{k-1}^{n-1}, S_{j-1}^{n-1}, S_{1}^{n}\right)+R_{x_{0}}\left(S_{1}^{n}, S_{j-1}^{n-1}, S_{k-1}^{n-1}, S_{1}^{n}\right)\right) \\
& =\sum_{k=2}^{n+1} \sum_{\substack{j=2 \\
j \neq k}}^{n+1} R_{x_{0}}\left(S_{1}^{n}, S_{k-1}^{n-1}, S_{j-1}^{n-1}, S_{1}^{n}\right)=-\sum_{k=2}^{n+1} R_{x_{0}}\left(S_{1}^{n}, S_{k-1}^{n-1}, S_{k-1}^{n-1}, S_{1}^{n}\right),
\end{aligned}
$$


and

$$
\begin{aligned}
\sum_{2 \leq k<j \leq n+1}\left(R_{x_{0}}\left(S_{1}^{n}, S_{k-1}^{n-1}, S_{k-1}^{n-1}, S_{j-1}^{n-1}\right)+R_{x_{0}}\left(S_{1}^{n}, S_{j-1}^{n-1}, S_{j-1}^{n-1}, S_{k-1}^{n-1}\right)\right) & =\sum_{j=2}^{n+1} \sum_{\substack{k=2 \\
k \neq j}}^{n+1} R_{x_{0}}\left(S_{1}^{n}, S_{j-1}^{n-1}, S_{j-1}^{n-1}, S_{k-1}^{n-1}\right) \\
& =-\sum_{j=2}^{n+1} R_{x_{0}}\left(S_{1}^{n}, S_{j-1}^{n-1}, S_{j-1}^{n-1}, S_{j-1}^{n-1}\right)=0 .
\end{aligned}
$$

Hence, we can compute

$$
\begin{aligned}
\sum_{1 \leq k<j \leq n+1} \kappa\left(S_{k}^{n}, S_{j}^{n}\right) & =\sum_{j=2}^{n+1} \kappa\left(S_{1}^{n}, S_{j}^{n}\right)+\sum_{2 \leq k<j \leq n+1} \kappa\left(S_{k}^{n}, S_{j}^{n}\right) \\
& =\sum_{j=2}^{n+1} \kappa\left(S_{1}^{n},-\frac{1}{n} S_{1}^{n}+\frac{\sqrt{n^{2}-1}}{n} S_{j-1}^{n-1}\right) \\
& +\sum_{2 \leq k<j \leq n+1} \kappa\left(-\frac{1}{n} S_{1}^{n}+\frac{\sqrt{n^{2}-1}}{n} S_{k-1}^{n-1},-\frac{1}{n} S_{1}^{n}+\frac{\sqrt{n^{2}-1}}{n} S_{j-1}^{n-1}\right) \\
& =\frac{n^{2}-1}{n^{2}} \sum_{j=2}^{n+1} \kappa\left(S_{1}^{n}, S_{j-1}^{n-1}\right)-2 \frac{n^{2}-1}{n^{4}} \sum_{2 \leq k<j \leq n+1} R_{x_{0}}\left(S_{1}^{n}, S_{k-1}^{n-1}, S_{j-1}^{n-1}, S_{1}^{n}\right) \\
& +\frac{n^{2}-1}{n^{4}} \sum_{2 \leq k<j \leq n+1}\left[\kappa\left(S_{1}^{n}, S_{j-1}^{n-1}\right)+\kappa\left(S_{1}^{n}, S_{k-1}^{n-1}\right)\right] \\
& -\frac{\left(n^{2}-1\right)^{3 / 2}}{n^{4}} \sum_{2 \leq k<j \leq n+1}\left[2 R_{x_{0}}\left(S_{1}^{n}, S_{j-1}^{n-1}, S_{j-1}^{n-1}, S_{k-1}^{n-1}\right)+2 R_{x_{0}}\left(S_{1}^{n}, S_{k-1}^{n-1}, S_{k-1}^{n-1}, S_{j-1}^{n-1}\right)\right] \\
& +\frac{\left(n^{2}-1\right)^{2}}{n^{4}} \sum_{2 \leq k<j \leq n+1} \kappa\left(S_{k-1}^{n-1}, S_{j-1}^{n-1}\right) .
\end{aligned}
$$

Inserting (2.27) and (2.28), and applying Lemma 2.7 with $p=n-1$, we get

$$
\begin{aligned}
\sum_{1 \leq k<j \leq n+1} \kappa\left(S_{k}^{n}, S_{j}^{n}\right) & =\frac{(n+1)^{2}}{n^{2}} \sum_{j=2}^{n+1} \kappa\left(S_{1}^{n}, S_{j-1}^{n-1}\right)+\frac{\left(n^{2}-1\right)^{2}}{n^{4}} \sum_{2 \leq k<j \leq n+1} \kappa\left(S_{k-1}^{n-1}, S_{j-1}^{n-1}\right) \\
& =\frac{(n+1)^{2}}{n^{2}}\left[\sum_{j=1}^{n-1} \operatorname{Sect}_{\chi_{0}}\left(e_{n} \wedge e_{j}\right)+\frac{(n-1)^{2}}{n^{2}} \sum_{2 \leq k<j \leq n+1} \kappa\left(S_{k-1}^{n-1}, S_{j-1}^{n-1}\right)\right]
\end{aligned}
$$

This latter, together with (2.25), proves Lemma 2.6.

\section{A possible generalization}

For real positive $p$, one could also define the $p^{\text {th }}$ order $q$-extent of a metric space $(X, d)$ as

$$
\mathrm{xt}_{q}^{(p)}(X):=\left(\begin{array}{c}
q \\
2
\end{array}\right)^{-1} \sup _{\left(x_{1}, \ldots, x_{q}\right) \in X^{q}} \sum_{1 \leq i<j \leq q}\left(d_{X}\left(x_{i}, x_{j}\right)\right)^{p} .
$$

It turns out that for $1 \leq p<2$, Theorem 1.1 and Corollary 1.2 generalize to $\mathrm{xt}_{q}^{(p)}(X)$. Namely one has

Theorem 2.8. Let $(M, g)$ be a smooth $n$-dimensional Riemannian manifold and $x \in \operatorname{int}(M)$. Then

$$
\mathrm{xt}_{n+1}^{(p)}\left(\bar{B}_{\varepsilon}^{M}(x)\right)=\varepsilon^{p} \mathrm{xt}_{n+1}^{(p)}\left(\bar{B}_{1}^{\mathbb{R}^{n}}\right)-\frac{p}{12 n^{2}}\left(\frac{2(n+1)}{n}\right)^{p / 2} \operatorname{Scal}_{g}(x) \varepsilon^{p+2}+\mathcal{O}\left(\varepsilon^{p+3}\right) .
$$


Corollary 2.9. Let $(M, g)$ be a smooth $n$-dimensional Riemannian manifold and $x \in \operatorname{int}(M)$. Then

$$
\operatorname{Scal}_{g}(x)=\lim _{\varepsilon \rightarrow 0} \frac{12 n^{2}}{p \varepsilon^{2}}\left(1-\frac{\mathrm{xt}_{n+1}^{(p)}\left(\bar{B}_{\varepsilon}^{M}(x)\right)}{\mathrm{xt}_{n+1}^{(p)}\left(\bar{B}_{\varepsilon}^{\mathbb{R}^{n}}\right)}\right) .
$$

These results can be proved essentially as the case $p=1$ treated above. The main difference is the proof of the stability result stated as Proposition 2.2. Namely, one has to replace relation (2.4) with the inequality

$$
\sum_{1 \leq k<j \leq n+1}\left|P_{\varepsilon, k}-P_{\varepsilon, j}\right|^{p} \leq\left(\frac{n(n+1)}{2}\right)^{1-p / 2}\left[(n+1)^{2}-(n+1)^{2}|G|^{2}-(n+1) \sum_{k=1}^{n+1}\left(1-\left|P_{\varepsilon, k}\right|^{2}\right)\right]^{p / 2} \mathcal{\varepsilon},
$$

where

$$
\mathcal{E}:=\left[1-\left(1-\frac{p}{2}\right) \sum_{1 \leq k<j \leq n+1}\left|\frac{\left|P_{\varepsilon, k}-P_{\varepsilon, j}\right|}{\left(\sum_{1 \leq k<j \leq n+1}\left|P_{\varepsilon, k}-P_{\varepsilon, j}\right|^{2}\right)^{1 / 2}}-\sqrt{\frac{2}{n(n+1)}}\right|^{2}\right] .
$$

This latter can be obtained using a quantitative version of Hölder inequality; see for instance [1, Theorem 2.2].

Note that, for $p \geq 2$, regular $(n+1)$-simplexes are no more the (unique) $(n+1)$-extenders of Euclidean balls, so that the proof fails to work in this case.

\section{Local extent in metric spaces}

The curvature of metric spaces is the object of an active research field. Since the seminal work by Alexandrov, a notion of metric sectional curvature upper (resp. lower) bounded is provided. For later purposes, we recall that a locally compact metric space $(X, d)$, whose metric $d$ is intrinsic, is a space of curvature bounded above (resp. below) by $k$ in the sense of Alexandrov if in some neighborhood of each point the following holds:

For every geodesic triangle $\Delta a b c$ with vertices $a, b$ and $c$ in $X$ and every point $d \in[a c]$, one has $|d b| \leq|\bar{d} \bar{b}|$ (resp. $|d b| \geq|\bar{d} \bar{b}|$ ) where $\bar{d}$ is the point on the side $[\bar{a} \bar{c}]$ of a comparison triangle $\Delta \bar{a} \bar{b} \bar{c}$ such that $|a d|=|\bar{a} \bar{d}|$. Here a comparision triangle is a triangle of vertices $\bar{a}, \bar{b}$ and $\bar{c}$ in the $k$-plane $S_{k}^{2}$ (i.e. the 2-dimensional simply connected space of constant curvature $k$ ) satisfying $|a b|=|\bar{a} \bar{b}|,|a c|=|\bar{a} \bar{c}|$ and $|b c|=|\bar{b} \bar{c}|$. Other equivalent definitions can be found for instance in [4].

In the last decades several possible notions of Ricci curvature bounds for (measure) metric spaced have been proposed and successfully investigated.

On the other hand, the aim for a metric notion of scalar curvature is much more recent and seems more difficult; see for instance $[13,14,23]$ and references therein. Specific answers to this problem have been given in particular context; see for instance [17, 19] for Alexandrov surfaces and [5, 6] for Alexandrov definable sets in $o$-minimal structures.

Here we propose the following definition

Definition 3.1. Let $(X, d)$ be a locally compact metric space and $x \in \operatorname{int}(X)$. We define the (n-dimensional) scalar curvature of $X$ at $x$ as

$$
\operatorname{Scal}_{d}^{(n)}(x)=\lim _{\varepsilon \rightarrow 0} \frac{12 n^{2}}{\varepsilon^{2}}\left\{1-\frac{\mathrm{xt}_{n+1}\left(\bar{B}_{\varepsilon}^{X}(x)\right)}{\mathrm{xt}_{n+1}\left(\bar{B}_{\varepsilon}^{\mathbb{R}^{n}}(x)\right)}\right\}
$$

whenever the limit exists.

Note that, in general, the scalar curvature of a metric space is not expected to be point-wise defined (consider for instance the singular part of a polyhedral space). Accordingly, it could be interesting to have bounds on the scalar curvaure. 
Definition 3.2. Let $(X, d)$ be a locally compact metric space. We say that $X$ has (n-dimensional) scalar curvature greater or equal to $k \in \mathbb{R}$ at $x \in X$, and we write $\mathrm{Scal}_{d}^{(n)}(x) \geq k$, if

$$
\liminf _{\varepsilon \rightarrow 0} \frac{12 n^{2}}{\varepsilon^{2}}\left\{1-\frac{\mathrm{xt}_{n+1}\left(\bar{B}_{\varepsilon}^{X}(x)\right)}{\mathrm{xt}_{n+1}\left(\bar{B}_{\varepsilon}^{\mathbb{R}^{n}}(x)\right)}\right\} \geq k .
$$

Similarly, we say that $X$ has (n-dimensional) scalar curvature smaller or equal to $k \in \mathbb{R}$ at $x \in X$, and we write $\mathrm{Scal}_{d}^{(n)}(x) \leq k$, if

$$
\limsup _{\varepsilon \rightarrow 0} \frac{12 n^{2}}{\varepsilon^{2}}\left\{1-\frac{\mathrm{xt}_{n+1}\left(\bar{B}_{\varepsilon}^{X}(x)\right)}{\mathrm{xt}_{n+1}\left(\bar{B}_{\varepsilon}^{\mathbb{R}^{n}}(x)\right)}\right\} \leq k .
$$

According to Lemma 2.1 below, in case $k=0$ the definition above specifies as follows. A metric space $X$ has nonnegative scalar curvature at $x$ if

$$
\limsup _{\varepsilon \rightarrow 0} \varepsilon^{-1} \mathrm{xt}_{n+1}\left(\bar{B}_{\varepsilon}^{X}(x)\right) \leq \sqrt{2 \frac{n+1}{n}},
$$

while $X$ has nonpositive scalar curvature at $x$ if

$$
\liminf _{\varepsilon \rightarrow 0} \varepsilon^{-1} \mathrm{xt}_{n+1}\left(\bar{B}_{\varepsilon}^{X}(x)\right) \geq \sqrt{2 \frac{n+1}{n}} .
$$

Thanks to Theorem 1.1 and the subsequent discussion, the above definitions of (bounds on the) $n$ dimensional scalar curvature are clearly consistent with the classical one when the underlying space is a Riemannian manifold. Moreover, they are also consistent with the curvature bounds in the sense of Alexandrov.

Recall that finite dimensional Alexandrov spaces with a lower curvature bound have a natural notion of dimension (in fact, all the reasonable notions of dimension coincide, and the dimension is a positive integer; see [4, Chapter 10]). Accordingly, as in the Riemannian setting one expects the following result, which is in fact a direct consequence of [4, Proposition 10.6.10].

Theorem 3.3. Let $(X, d)$ be a $n$-dimensional $C B B(k)$ Alexandrov space, for some $k \in \mathbb{R}$, then for all $x \in X$ and $\epsilon>0$,

$$
\mathrm{xt}_{n+1}\left(\bar{B}_{\varepsilon}^{X}(x)\right) \leq \mathrm{xt}_{n+1}\left(\bar{B}_{\varepsilon}^{S_{k}^{n}}\right),
$$

where $S_{k}^{n}$ is the simply connected $n$-dimensional space form of constant curvature $k$. In particular

$$
\operatorname{Scal}_{d}^{(n)}(x) \geq k n(n-1) \text {. }
$$

Let $\Delta_{k}^{n+1}(\epsilon)$ be the unique (up to isometries) regular $(n+1)$-simplex inscribed in $\bar{B}_{\varepsilon}^{S_{k}^{n}}$ (that is, with totally geodesic faces in $S_{k}^{n}$ ). Note that $\Delta_{0}^{n+1}(1)=\mathcal{S}^{n+1}$. Similarly to an analogous rigidity result for the packing radius (see [11, Lemma 3.3]), we have the following

Proposition 3.4. Let $(X, d)$ be a n-dimensional $C B B(k)$ Alexandrov space, for some $k \in \mathbb{R}$. Suppose that for some $x \in X$ and $\epsilon>0$ (with $\epsilon \leq \frac{\pi}{4 \sqrt{k}}$ if $k>0$ ),

$$
\mathrm{xt}_{n+1}\left(\bar{B}_{\varepsilon}^{X}(x)\right)=\mathrm{xt}_{n+1}\left(\bar{B}_{\varepsilon}^{S_{k}^{n}}\right) .
$$

Then an isometric copy of $\Delta_{k}^{n+1}(\epsilon)$ with totally geodesic interior is inscribed in $\bar{B}_{\varepsilon}^{X}(x)$. In particular, there exists $\delta_{0}$ depending on $\epsilon$, $k$ and $n$ such that $\bar{B}_{\delta}^{X}(x)$ is isometric to $\bar{B}_{\delta}^{S_{k}^{n}}$ for every $0<\delta \leq \delta_{0}$.

Remark 3.5. As for [11, Lemma 3.3], Proposition 3.4 is sharp, in the sense that in general we can not expect $\bar{B}_{\varepsilon}^{X}(x)$ and $\bar{B}_{\varepsilon}^{S_{k}^{n}}$ to be isometric. A trivial counterexample is given by the Alexandrov space $\Delta_{k}^{n+1}(\epsilon)$, endowed with the metric induced by $S_{k}^{n}$.

The bound on $\epsilon$ is given by the fact that regular simplexes are no more extenders of $\bar{B}_{\varepsilon}^{S_{k}^{n}}$ for $\epsilon$ close to $\frac{\pi}{2 \sqrt{k}}$ (although they continue being packers). 
Proof. Let $\left\{Q_{j}\right\}_{j=1}^{n+1}$ be a $(n+1)$-extender for $\bar{B}_{\varepsilon}^{X}(x)$. Let $f: X \rightarrow S_{k}^{n}$ be the noncontracting map given by [4, Proposition 10.6.10], and define $\left\{P_{j}\right\}_{j=1}^{n+1} \subset \bar{B}_{\varepsilon}^{S_{k}^{n}}(x)$ as $P_{j}=f\left(Q_{j}\right)$. By the non-contractivity of $f$, it holds

$$
d_{S_{k}^{n}}\left(P_{j}, P_{k}\right) \geq d\left(Q_{j}, Q_{k}\right)
$$

for every $k$ and $j$. In particular

$$
\begin{aligned}
\left(\begin{array}{c}
n+1 \\
2
\end{array}\right) \mathrm{xt}_{n+1}\left(\bar{B}_{\varepsilon}^{X}(x)\right) & =\sum_{1 \leq j<k \leq n+1} d\left(Q_{j}, Q_{k}\right) \\
& \leq \sum_{1 \leq j<k \leq n+1} d_{S_{k}^{n}}\left(P_{j}, P_{k}\right) \\
& \leq\left(\begin{array}{c}
n+1 \\
2
\end{array}\right) \mathrm{xt}_{n+1}\left(\bar{B}_{\varepsilon}^{S_{k}^{n}}\right) .
\end{aligned}
$$

Equality in (3.2), together with (3.3), implies that

$$
d_{S_{k}^{n}}\left(P_{j}, P_{k}\right)=d\left(Q_{j}, Q_{k}\right)
$$

for every $k$ and $j$ and that $\left\{P_{j}\right\}_{j=1}^{n+1}$ is a $(n+1)$-extender for $\bar{B}_{\varepsilon}^{S_{k}^{n}}$, which is hence given by $\Delta_{k}^{n+1}(\epsilon)$; see [11, page 9].

According to the characterization given in [11, page 19], $\left\{P_{j}\right\}_{j=1}^{n+1}$ is also a $(n+1)$-packer of $\bar{B}_{\varepsilon}^{S_{k}^{n}}$. From (3.4), we deduce that $\operatorname{pack}_{n+1}\left(\bar{B}_{\varepsilon}^{X}\right) \geq \operatorname{pack}_{n+1}\left(\bar{B}_{\varepsilon}^{S_{k}^{n}}\right)$. By angle comparison, this inequality is in fact an equality, as observed also in [11, (3.1)]. By the proof of [11, Lemma 2.3], we get that $\bar{B}_{\varepsilon}^{X}$ contains an isometric copy of $\Delta_{k}^{n+1}(\epsilon)$ with totally geodesic interior and vertexes given by $\left\{Q_{j}\right\}_{j=1}^{n+1}$.

In the assumption of an upper bound on the curvature, there is not a general natural notion of dimension. However, one can still introduce a geometric dimension $\operatorname{GeomDim}(X)$ of a CBA space $X$, which is defined as the smallest function defined on CBA spaces such that a) $\operatorname{GeomDim}(X)=0$ whenever $X$ is a discrete space, and b) $\operatorname{GeomDim}(X) \geq 1+\operatorname{GeomDim}\left(\Sigma_{p} X\right)$ for every $p \in X, \Sigma_{p} X$ being the space of directions; see [16]. B. Kleiner proved in particular that $\operatorname{GeomDim}(X)$ is equal to the topological dimension of the space. Moreover, whenever $\operatorname{GeomDim}(X)<+\infty$, it holds that $\operatorname{Geom\operatorname {Dim}}(X)$ is the greatest integer $q$ such that there is an isometric embedding of the standard unit sphere $\mathbb{S}^{n-1} \subset \mathbb{R}^{n}$ into $\Sigma_{p} X$ for some $p \in X$. By the definition of the space of directions and by the monotonicity condition for upper curvature bounds on metric space (see [4, Section 4.3.1]), one easily gets

Proposition 3.6. Let $(X, d)$ be a $C B A(k)$ Alexandrov space. Suppose that there is an isometric embedding of the standard unit sphere $\mathbb{S}^{n-1} \subset \mathbb{R}^{n}$ into $\Sigma_{X} X$ for some $x \in X$. Then

$$
\operatorname{Scal}_{d}^{(n)}(x) \leq k n(n-1) .
$$

\section{Curvature measure of $C B B(k)$ surfaces}

In this section we focus on surfaces with lower bounded curvature. Let $(S, d)$ be a 2-dimensional topological surface, whose metric is $C B B(k)$ for some $k \in \mathbb{R}$. $(S, d)$ is in particular a surface of bounded integral curvature (in abbreviated form BIC surface) so that it supports a well-defined curvature measure $\omega$, see for instance [22], [3] or the survey [24]. This latter measure constitutes a generalization of the Riemannian curvature of a surface to the non-smooth setting, i.e. $d \omega=\operatorname{Sect}_{g} d A_{g}=\frac{1}{2}$ Scal $_{g} d A_{g}$ whenever the distance $d$ of $(S, d)$ is induced by a smooth Riemannian metric $g$ on $S$. In fact $\omega$ can be defined as the weak limit (in the sense of measures) of the integral of the sectional curvature of an approximating sequence of smooth metrics. In [19], Y. Machigashira studied the regular part $\omega_{\text {reg }}$ of the curvature measure of a $C B B(k)$ surface with respect to the 
2-dimensional Hausdorff measure (restricted to Borel sets). He introduced the (almost everywhere defined) Gaussian curvature function $G$ on $S$ by

$$
G(x)=\inf _{d>0} \underline{G_{d}}(x) ; \quad \underline{G_{d}}(x):=\liminf _{\Delta \rightarrow\{x\}, x \in \Delta, \Delta \in U_{d}} \frac{e(\Delta)}{\operatorname{Area}(\Delta)}
$$

and proved that $\omega_{\text {reg }}(E)=\int_{E} G(x) d \mathcal{H}^{2}$ for every suitably measurable set $E \subset S$ (see [19] for details). The liminf in (4.1) is computed with respect to geodesic triangles converging to the point $x$, containing $x$ in their interior, and with interior angles greater than $d>0$. Moreover the excess $e(\Delta)$ of the geodesic triangle $\Delta$ is defined so that $e(\Delta)+\pi$ is the sum of the interior angles of $\Delta$.

In the following proposition, we are going to prove that the characterization of the scalar curvature via the local extent holds on a $C B B(k)$ surface at normal points, which are roughly points at which one has a second order differentiable structure (see [25] for a rigorous definition of normal point).

Proposition 4.1. Let $(S, d)$ be a $C B B(k)$ surface, $k \in \mathbb{R}$. Then for $\mathcal{H}^{2}$-a.e. $x \in S$ it holds

$$
G(x)=\frac{1}{2} \operatorname{Scal}_{d}^{(2)}(x)
$$

Proof. Since $C B B(1)$ surfaces are in particular $C B B(-1)$, up to a rescaling we can suppose without loss of generality that $k=-1$. According to a well-known result by Alexandrov, [2, Section XII.2], each point in $(S, d)$ has a neighborhood isometric to the boundary of a convex set $K$ in $\mathbb{H}^{3}$. By [25, Proposition 6] a.e. point in $\partial K$ is normal. At every normal point $q \in \partial K$ the following hold: for $\epsilon \ll 1$, there exists a biLipschitz homeomorphism $\Phi_{\varepsilon}$ from the metric ball $B_{\epsilon}(q) \subset \partial K$ onto an open set $\Phi_{\varepsilon}\left(B_{\epsilon}(q)\right)=: U_{\epsilon} \subset S_{G(q)}^{2}$ contained in the simply connected smooth surface $S_{G(q)}^{2}$ of constant sectional curvature $G(q)$, so that the Lipschitz constants $L(\Phi)$ and $L\left(\Phi^{-1}\right)$ are smaller than $(1+h(\epsilon))$, where the function $h$ is such that $\varepsilon^{-2} h(\varepsilon) \rightarrow 0$ as $\epsilon \rightarrow 0$, [25, Corollary 9 and Theorem 14].

In particular for every couple of points $x$ and $y$ in $(S, d)$ such that $d(x, q) \leq \varepsilon$ and $d(y, q) \leq \varepsilon$, one has $\Phi_{\varepsilon}(x), \Phi_{\varepsilon}(y) \in \bar{B}_{\varepsilon+\varepsilon h(\varepsilon)} \subset S_{G(q)}^{2}$. Accordingly,

$$
\begin{aligned}
\operatorname{xt}_{3}\left(\bar{B}_{\varepsilon}^{S}(q)\right) & =\frac{1}{3} \sup _{\left(x_{1}, x_{2}, x_{3}\right) \in S^{3}} \sum_{1 \leq i<q \leq 3} d\left(x_{i}, x_{j}\right) \\
& \leq \frac{1}{3}(1+h(\varepsilon)) \sup _{\left(x_{1}, x_{2}, x_{3}\right) \in S^{3}} \sum_{1 \leq i<q \leq 3} d_{S_{G(q)}^{2}}\left(\Phi_{\varepsilon}\left(x_{i}\right), \Phi_{\varepsilon}\left(x_{j}\right)\right) \\
& \leq(1+h(\varepsilon)) \mathrm{xt}_{3}\left(\bar{B}_{\varepsilon+\varepsilon h(\varepsilon)}^{S_{G(q)}^{2}}\right),
\end{aligned}
$$

so that

$$
\begin{aligned}
\frac{12 n^{2}}{\varepsilon^{2}}\left\{1-\frac{\mathrm{xt}_{3}\left(\bar{B}_{\varepsilon}^{S}(q)\right)}{\mathrm{xt}_{3}\left(\bar{B}_{\varepsilon}^{\mathbb{R}^{n}}(x)\right)}\right\} & \geq \frac{12 n^{2}}{\varepsilon^{2}}\left\{1-\frac{(1+h(\varepsilon)) \mathrm{xt}_{3}\left(\bar{B}_{\varepsilon}^{S_{G(q)}^{2}}\right)}{\mathrm{xt}_{3}\left(\bar{B}_{\varepsilon}^{\mathbb{R}^{n}}(x)\right)}\right\} \\
& \geq \frac{12 n^{2}}{\varepsilon^{2}}\left\{1-\frac{\mathrm{xt}_{3}\left(\bar{B}_{\varepsilon}^{S_{G(q)}^{2}}\right)}{\mathrm{xt}_{3}\left(\bar{B}_{\varepsilon}^{\mathbb{R}^{n}}(x)\right)}-h(\varepsilon) \sqrt{\frac{2 n}{n+1}}\right\} .
\end{aligned}
$$

In particular, by Definition 3.1, $\mathrm{Scal}_{S}^{(2)}(q) \geq 2 G(q)$. Exchanging the roles of $S$ and $S_{q}$ in (4.3) and (4.4) gives the converse inequality $\operatorname{Scal}_{S}^{(2)}(q) \leq 2 G(q)$.

\section{Towards scalar curvature measure}

Consider a metric measure space $(X, d, \mu)$, that is a metric space $(X, d)$ endowed with a positive Radon measure $\mu$. Kapovitch, Lytchak and Petrunin recently proposed a notion of metric scalar curvature $v$ on $(X, d, \mu)$ 
which they called metric-measure curvature. Namely, for $x \in X$ define $b_{r}(x):=\mu\left(B_{r}(x)\right)$ and introduce the deviation measure $v_{r}$ on $X$, absolutely continuous with respect to $\mu$, given by

$$
v_{r}=\left(1-\frac{b_{r}}{\operatorname{Vol}\left(B_{r}^{\mathbb{R}^{n}}(x)\right)}\right) \mu .
$$

This is primarly motivated by the fact that, when $X=(M, g)$ is a Riemannian manifold endowed with Riemannian distance $d=d_{g}$ and volume $\mu=$ Vol $_{g}$, according to (1.1) the one-parameter family $\left\{v_{r} / r^{2}\right\}_{r>0}$ converges as a measure to $\frac{1}{6(n+2)} \mathrm{Scal}_{g} d \mathrm{Vol}_{g}$ as $r \rightarrow 0$. Accordingly, one expects the limit measure

$$
v:=\lim _{r \rightarrow 0} v_{r} / r^{2}
$$

whenever it exists, to be a good candidate to replace the scalar curvature on measure metric spaces. In [17] it is in particular proven that the family of measure $v_{r} / r^{2}$ is uniformly bounded on surfaces with bounded integral curvature, so that it exists a (a priori non unique) metric-measure curvature $v$. Note that unfortunately $v$ does not coincide in general with the natural intrinsic curvature measure of the surface; see [17, Exemple 1.14].

In this section we adapt the construction of [17] to define an extent-curvature measure and a boundaryextent-curvature-measure which intend to generalize the usual scalar curvature (measure) of a Riemannian manifold to non-necessarily smooth spaces using (1.3) or (1.4) instead of (1.1). As it is the case for the metric measure curvature, we will see that extent-curvature measures and boundary-extent-curvature measures exist on BIC surfaces.

In the following, let $(S, d)$ be a surface of bounded integral curvature and consider its area measure (which coincides with the two-dimensional Hausdorff measure $\mathcal{H}^{2}$ ). For any fixed scale $r$, consider the deviation measure $e_{r}$ on $X$, absolutely continuous with respect to $\mathcal{H}^{2}$, given by

$$
e_{r}:=\left(1-\frac{\mathrm{xt}_{3}\left(\bar{B}_{r}^{d}(x)\right)}{\mathrm{xt}_{3}\left(\bar{B}_{r}^{\mathbb{R}^{n}}\right)}\right) \mathcal{H}^{2} .
$$

We will say that $(S, d)$ has locally finite extent curvature if the family of measures $\left\{e_{r} / r^{2}\right\}_{0<r \leq 1}$ is uniformly bounded. Whenever a limit $\lim _{r \rightarrow 0} e_{r} / r^{2}$ exists, it is called extent curvature measure. Similarly, we can define

$$
\partial e_{r}:=\left(1-\frac{\partial \mathrm{xt}_{3}\left(\bar{B}_{r}^{d}(x)\right)}{\partial \mathrm{xt}_{3}\left(\bar{B}_{r}^{\mathbb{R}^{n}}\right)}\right) \mathcal{H}^{2}
$$

and say that $(S, d)$ has locally finite boundary extent curvature if the family of measures $\left\{\partial e_{r} / r^{2}\right\}_{0<r \leq 1}$ is uniformly bounded. Whenever a limit $\lim _{r \rightarrow 0} \partial e_{r} / r^{2}$ exists, it is called boundary extent curvature measure.

We have the following result.

Theorem 5.1. Let $(S, d)$ be a surface with bounded integral curvature.

1. $(S, d)$ has locally finite extent curvature. In particular there exists at least one extent curvature measure on $(S, d)$.

2. $(S, d)$ has locally finite boundary extent curvature. In particular there exists at least one boundary extent curvature measure on $(S, d)$.

Proof. The proof is essentially the same as the proof of [17, Theorem 4.2]. We will sketch the relevant changes for completeness.

The main ingredient is the analogue of [17, Lemma 4.1]

Lemma 5.2. There exists some $\delta_{0}>0$ with the following property. Let $S$ be a surface with bounded integral curvature and let $\omega$ be its curvature measure. Let $x \in X$ be a point and let $r>0$ be such that $\bar{B}_{r}^{d}(x)$ is compact, $|\omega|\left(B_{r}^{d}(x)\right)<\delta_{0}$ and $\bar{B}_{r}^{d}(x) \subset U$ where $U$ is an open subset of $S$ homeomorphic to $\mathbb{R}^{2}$. Then

$$
\left|1-\frac{\mathrm{xt}_{3}\left(\bar{B}_{r}^{d}(x)\right)}{\mathrm{xt}_{3}\left(\bar{B}_{r}^{\mathbb{R}^{2}}\right)}\right| \leq 3|\omega|\left(B_{r}^{d}(x)\right)
$$


and

$$
\left|1-\frac{\partial \mathrm{xt}_{3}\left(\bar{B}_{r}^{d}(x)\right)}{\partial \mathrm{xt}_{3}\left(\bar{B}_{r}^{\mathbb{R}^{2}}\right)}\right| \leq 3|\omega|\left(B_{r}^{d}(x)\right) .
$$

Proof. To prove (5.1), it suffices to prove that for all $0<s<r$

$$
\left|1-\frac{\mathrm{xt}_{3}\left(\bar{B}_{s}^{d}(x)\right)}{\mathrm{xt}_{3}\left(\bar{B}_{s}^{\mathbb{R}^{2}}\right)}\right| \leq 3|\omega|\left(B_{r}^{d}(x)\right) .
$$

Moreover it is enough to check this latter inequality on polyhedral metrics homeomorphic to $\mathbb{R}^{2}$. In fact, let $s<s^{\prime}<t$. According to [22, Theorem 4.3] we take a polyhedral sequence $\left\{d_{k}\right\}$ of metrics on $S$ approaching $d$ uniformly and tamely, see Theorm 8.4.3 and the subsequent discussion [22], or [3, Theorem 7 of Chapter VII]. Without loss of generality let

$$
\sup _{y, y^{\prime} \in U}\left|d\left(y, y^{\prime}\right)-d_{k}\left(y, y^{\prime}\right)\right|<\frac{1}{k} .
$$

Suppose that

$$
\left|1-\frac{\mathrm{xt}_{3}\left(\bar{B}_{s}^{d_{k}}(x)\right)}{\mathrm{xt}_{3}\left(\bar{B}_{s}^{\mathbb{R}^{n}}\right)}\right| \leq 3|\omega|\left(B_{s^{\prime}}^{d_{k}}(x)\right)
$$

for any polyhedral metric $d_{k}$. Let $x_{j, k}, j=1,2,3$ be a 3-extender of $\bar{B}_{s}^{d_{k}}(x)$. Then $x_{j, k} \in B_{s+\frac{1}{k}}^{d}(x)$ and xt ${ }_{3}\left(\bar{B}_{s}^{d}(x)\right) \geq$ $\lim \sup _{k \rightarrow \infty} \mathrm{xt}_{3}\left(\bar{B}_{s}^{d_{k}}(x)\right)$. Similarly, if $\left\{y_{j}\right\}_{j=1,2,3}$ is a 3-extender of $\bar{B}_{S}^{d}(x)$, then $y_{j} \in B_{s+1 / k}^{d_{k}}(x)$ so that $x t_{3}\left(\bar{B}_{s}^{d}(x)\right) \leq$ $\liminf _{k \rightarrow \infty} \operatorname{xt}_{3}\left(\bar{B}_{s+1 / k}^{d_{k}}(x)\right)$.

Then the proof is the same as in [17], and it exploits the construction of an explicit completion of the local polyhedral surface to a global polyhedral BIC metric on $\mathbb{R}^{2}$ with controlled curvature, as well as the biLipschitz maps to $\mathbb{R}^{2}$ provided by [7].

To conclude, one has just to remark that on regions $\left(1+\delta^{\prime}\right)$-biLipschitz diffeomorphic to Euclidean open sets it holds

$$
\left(1+\delta^{\prime}\right)^{-2} \leq \frac{\mathrm{xt}_{3}\left(\bar{B}_{r}(x)\right)}{\mathrm{xt}_{3}\left(\bar{B}_{r}^{\mathbb{R}^{n}}\right)} \leq\left(1+\delta^{\prime}\right)^{2}
$$

for $r$ small enough (compare with [17, Section 2.4]).

The proof of (5.2) is essentially the same. One has just to keep in account that by definition $\mid \partial \mathrm{xt}_{3}\left(\bar{B}_{r}(x)\right)-$ $\mathrm{xt}_{3}\left(\bar{B}_{r}(x)\right) \mid<\eta$ whenever the three points realizing $\mathrm{xt}_{3}\left(\bar{B}_{r}(x)\right)$ are in $B_{r}(x) \backslash B_{r-\eta / 6}(x)$.

We prove (1), the proof of (2) being essentially the same. The result is local so that it suffices to prove it in a neighborhood of an arbitrary point $x_{0} \in S$. Let $X$ be a neighborhood of $x_{0}$ homeomorphic to $\mathbb{R}^{2}$ and satisfying $|\omega|\left(X \backslash\left\{x_{0}\right\}\right)<\min \left\{\delta_{0} ; 1 / 3\right\}, \delta_{0}$ being the positive constant in Lemma 5.2. Let $A \subset X$ compact. We want to prove that there exist an $\varepsilon>0$ and a constant $C=C(\epsilon)>0$ such that for all $0<r<\epsilon$ one has

$$
\left|e_{r}\right|(A) \leq C r^{2}
$$

First note that for any $x \in S$ and positive $s$, it holds $\mathrm{xt}_{3}\left(\bar{B}_{s}(x)\right) \leq 2 s$ and

$$
1 \geq 1-\frac{\mathrm{xt}_{3}\left(\bar{B}_{s}(x)\right)}{\mathrm{xt}_{3}\left(\bar{B}_{s}^{\mathbb{R}^{n}}\right)} \geq(1-2 \sqrt{3} / 3)>-1 .
$$

According to [17], up to take a smaller $\epsilon$ we have that for all $0<3 r<\epsilon$

$$
\mathcal{H}^{2}\left(B_{3 r}\left(x_{0}\right)\right)<\frac{9 r^{2}}{4 \epsilon} .
$$

Then

$$
\left|e_{r}\right|\left(A \cap B_{2 r}\left(x_{0}\right)\right) \leq\left|e_{r}\right|\left(B_{2 r}\left(x_{0}\right)\right) \leq \mathcal{H}^{2}\left(B_{3 r}\left(x_{0}\right)\right)<\frac{9 r^{2}}{4 \epsilon} .
$$


On the other hand, for $x \in X \backslash B_{r}\left(x_{0}\right)$ we have $|\omega|\left(B_{r}(x)\right)<\delta_{0}$. We can thus apply Lemma 5.2 and [17, Lemmas 2.1 and 4.1] to get

$$
\begin{aligned}
\left|e_{r}\right|\left(A \backslash B_{2 r}\left(x_{0}\right)\right) & \leq \int_{A \backslash B_{2 r}\left(x_{0}\right)} 3|\omega|\left(B_{r}(x)\right) d \mathcal{H}^{2}(x) \\
& \leq 3 \int_{A \backslash B_{r}\left(x_{0}\right)} \mathcal{H}^{2}\left(B_{r}(x)\right) d|\omega|(x) \\
& \leq 6 \pi r^{2}|\omega|\left(A \backslash B_{r}\left(x_{0}\right)\right) \\
& \leq 6 \pi \delta_{0} r^{2} .
\end{aligned}
$$

Thus $\left|e_{r}\right|(A) \leq\left(6 \pi \delta_{0}+9 / 4 \epsilon\right) r^{2}$, as claimed.

Acknowledgements: I would like to thank J. Bertrand for useful discussion on the subject. In particular, I am indebted to him for suggesting the approach to Proposition 4.1 via the local isometry between Alexandrov surfaces and convex surfaces in spaces of constant curvature, and for pointing me out the Alexandrov's theorem on the a.e. second differentiability of convex functions. Also, I'm grateful to A. Bernig for pointing out to me the paper [17], from which Section 5 arose.

This research has been conducted as part of the project Labex MME-DII (ANR11-LBX-0023-01). The author is members of the "Gruppo Nazionale per l'Analisi Matematica, la Probabilitá e le loro Applicazioni" (GNAMPA) of the Istituto Nazionale di Alta Matematica (INdAM).

\section{References}

[1] J. M. Aldaz. A stability version of Hölder's inequality. J. Math. Anal. Appl., 343(2):842-852, 2008.

[2] A. D. Alexandrov. A. D. Alexandrov selected works. Part II. Chapman \& Hall/CRC, Boca Raton, FL, 2006. Intrinsic geometry of convex surfaces, Edited by S. S. Kutateladze, Translated from the Russian by S. Vakhrameyev.

[3] A. D. Aleksandrov and V. A. Zalgaller. Intrinsic geometry of surfaces. Translated from the Russian by J. M. Danskin. Translations of Mathematical Monographs, Vol. 15. American Mathematical Society, Providence, R.I., 1967.

[4] Dmitri Burago, Yuri Burago, and Sergei Ivanov. A course in metric geometry, volume 33 of Graduate Studies in Mathematics. American Mathematical Society, Providence, RI, 2001.

[5] Andreas Bernig. Scalar curvature of definable Alexandrov spaces. Adv. Geom., 2(1):29-55, 2002.

[6] Andreas Bernig. Scalar curvature of definable CAT-spaces. Adv. Geom., 3(1):23-43, 2003.

[7] Mario Bonk and Urs Lang. Bi-Lipschitz parameterization of surfaces. Math. Ann., 327(1):135-169, 2003.

[8] Leo Brewin. Riemann normal coordinate expansions using Cadabra. Classical Quantum Gravity, 26(17):175017, $17,2009$.

[9] Sylvestre Gallot. Inégalités isopérimétriques et analytiques sur les variétés riemanniennes. Astérisque, (163-164):5-6, 31-91, 281 (1989), 1988. On the geometry of differentiable manifolds (Rome, 1986).

[10] Karsten Grove and Steen Markvorsen. Curvature, triameter, and beyond. Bull. Amer. Math. Soc. (N.S.), 27(2):261-265, 1992.

[11] Karsten Grove and Steen Markvorsen. New extremal problems for the Riemannian recognition program via Alexandrov geometry. J. Amer. Math. Soc., 8(1):1-28, 1995.

[12] Alfred Gray. The volume of a small geodesic ball of a Riemannian manifold. Michigan Math. J., 20:329-344 (1974), 1973.

[13] Misha Gromov. Dirac and Plateau billiards in domains with corners. Cent. Eur. J. Math., 12(8):1109-1156, 2014.

[14] Misha Gromov. A dozen problems, questions and conjectures about positive scalar curvature. arXiv:1710.05946, 2017.

[15] Paul Jung, Tucker McElroy, and Jason Samuels. The n-extent of $s^{3}(p, m)$. Electronic Journal of Undergraduate Mathematics, 1:1-11, 1995.

[16] Bruce Kleiner. The local structure of length spaces with curvature bounded above. Math. Z., 231(3):409-456, 1999.

[17] Vitali Kapovitch, Alexander Lytchak, and Anton Petrunin. Metric-measure boundary and geodesic flow on alexandrov spaces. arXiv:1705.04767, 2017.

[18] J. N. Lillington. Some extremal properties of convex sets. Math. Proc. Cambridge Philos. Soc., 77:515-524, 1975.

[19] Yoshiroh Machigashira. The Gaussian curvature of Alexandrov surfaces. J. Math. Soc. Japan, 50(4):859-878, 1998.

[20] Steenn Markvorsen. Curvature and shape. Zb. Rad. Mat. Inst. Beograd. (N.S.), 6(14):55-75, 1997. 11th Yugoslav Geometrical Seminar (Divčibare, 1996).

[21] Richard S. Palais. On the differentiability of isometries. Proc. Amer. Math. Soc., 8:805-807, 1957.

[22] Yu. G. Reshetnyak. Two-dimensional manifolds of bounded curvature. In Geometry, IV, volume 70 of Encyclopaedia Math. Sci., pages 3-163, 245-250. Springer, Berlin, 1993. 
[23] Christina Sormani. Scalar curvature and intrinsic flat convergence. In Measure Theory in Non-Smooth Spaces, pages 288338. De Gruyter Open, August 2017.

[24] Marc Troyanov. Les surfaces à courbure intégrale bornée au sens d’Alexandrov. Annual day of the SMF, pages 1-18, 2009.

[25] Giona Veronelli. Boundary structure of convex sets in the hyperbolic space. Monatshefte für Mathematik, (in press), 2018.

[26] DaGang Yang. On the topology of non-negatively curved simply connected 4-manifolds with discrete symmetry. Duke Math. J., 74(2):531-545, 1994. 